\title{
Das Institut für Sächsische Geschichte und Volkskunde e. V. in Dresden Bericht für das Jahr 2012*
}

von

\author{
ENNO BÜNZ
}

Die Schwerpunkte der Institutsarbeit im Berichtszeitraum sind an den vier gemeinsamen Vorhaben der Bereiche Geschichte und Volkskunde, neun Projekten des Bereichs Geschichte und elf des Bereichs Volkskunde ablesbar. Von den gemeinsamen Vorhaben der beiden Arbeitsbereiche des Instituts ist die „Entwicklung und Durchführung einer Konzeption für die Dauerausstellung zur Geschichte der Deutschen in den böhmischen Ländern" besonders hervorzuheben. Es basiert auf einer grenzüberschreitenden Kooperation und wird im Rahmen von Ziel-3 finanziert. Parallel zur Ausstellungsvorbereitung in Ústí nad Labem/Aussig konnten die drei Begleitpublikationen durch das ISGV vorgelegt werden.

Im Bereich Geschichte hat das Langzeitprojekt „Sächsische Biografie“ weiterhin gute Fortschritte gemacht. Gleiches gilt für die beiden Editionsvorhaben der Briefe der Herzogin Elisabeth von Sachsen (Fürstinnenkorrespondenzen der Reformationszeit) und des Urkundenbuchs der Stadt Dresden (Codex diplomaticus Saxoniae, Hauptteil II). Für das Drittmittelprojekt „Sächsisches Klosterbuch“, eine systematische Darstellung der 76 Klöster, Stifte und Kommenden im Gebiet des Freistaats Sachsen, konnten die meisten Beiträge eingeworben werden, doch ist die Finanzierung Ende des Jahres ausgelaufen. Durch die Vergabe der Promotionsförderung im Institut konnte mit der Arbeit an der Dissertation „Für Gott und Vaterland - Patriotismus und Militärdienst in Sachsen 1806 bis 1866/67“ ein neues historisches Forschungsprojekt begonnen werden.

Im Bereich Volkskunde sind bei den Langzeitvorhaben „Lebensgeschichtliches Archiv für Sachsen“, dessen neue Datenbanklösung nun weiter ausgebaut wird, und „Visuelle Quellen zur Volkskultur in Sachsen“ wieder gute Fortschritte erzielt worden. Im Rahmen des Drittmittelprojekts „Fremde - Heimat - Sachsen“ über Neubauern in Sachsen konnten sowohl die Interviewerhebung als auch die Archivreche abgeschlossen und mit der Präsentation von Ergebnissen im Rahmen einer Wanderausstellung begonnen werden. Das DFG-finanzierte Projekt „Das Auge des Arbeiters" über proletarische Amateurfotografie aus Sachsen ist erfolgreich abgeschlossen worden. Auch die Vorhaben „An der Elbe. Leben mit dem Fluss“ und „Neue Sichtweisen“ über das Aufleben der Aussichtsturm-Begeisterung in Sachsen Ende des 20. Jahrhunderts sind weiter vorangekommen.

Trotz einer zunehmend angespannten Haushaltslage, deren weitere Verschärfung die Arbeitsfähigkeit des Instituts nachhaltig beeinträchtigen würde, konnten im Berichtszeitraum nicht nur die laufenden Projekte weiter fortgesetzt werden, sondern das Institut und seine Mitarbeiter haben sich auch durch Tagungen und Vorträge, Lehrtätigkeit an Universitäten, Beratungstätigkeit in Gremien und Kommissionen, Buchvorstellungen und andere Veranstaltungen der interessierten Öffentlichkeit präsentiert.

* Siehe den letzten Bericht in: NASG 83 (2012), S. 273-279. 
Angesichts des insgesamt begrenzten finanziellen und personellen Rahmens leistet das ISGV als Dienstleister im Lande und darüber hinaus eine viel beachtete Arbeit, die auch von den die Institutsarbeit begleitenden Gremien positiv anerkannt wird.

Die im Berichtszeitraum durchgeführten fünf Tagungen galten der Adelsgeschichte in der Frühen Neuzeit, den Problemen der Präsentation ethnischer und nationaler Minderheiten im Museum, der vorreformatorischen Frömmigkeit, der Kulturlandschaft Elbe sowie der monastischen Geschichte Sachsens. Diese Veranstaltungen standen zumeist im Zusammenhang mit laufenden Arbeitsvorhaben und wurden zum Teil in Verbindung mit anderen Einrichtungen durchgeführt. In diesem Zusammenhang ist $\mathrm{zu}$ betonen, dass das ISGV mittlerweile auch ein vielgefragter Veranstaltungspartner ist, aufgrund der begrenzten finanziellen und personellen Ressourcen aber gar nicht alle Kooperationsanfragen erfüllen kann.

Von den Ausstellungsvorhaben, an deren Konzeption das ISGV mitwirkt, ist neben dem oben genannten Museumsprojekt zur Geschichte der Deutschen in den böhmischen Ländern auf weitere Vorhaben hinzuweisen, die 2012 vorbereitet wurden. Themen sind die vorreformatorische Frömmigkeitsgeschichte, die sächsische Adelskultur und der Friede von Hubertusburg 1763. Das ISGV ist ebenso in die Vorbereitung der 1. Brandenburgischen Landesausstellung eingebunden, die unter dem Titel „Preußen und Sachsen. Szenen einer Nachbarschaft" 2014 in Doberlug-Kirchhain stattfinden wird. Seitens des ISGV konnte die konzeptionelle Mitarbeit am „Haus der Archäologie und Geschichte Sachsens" in Chemnitz im Berichtsjahr abgeschlossen werden; die Einrichtung soll 2014 als Staatliches Museum für Archäologie eröffnet werden, wobei der unverbindliche Untertitel „Kulturen entdecken - Geschichte verstehen“ zumindest noch ansatzweise an die maßgebliche Planungsarbeit des ISGV erinnern wird.

Auf mehreren Veranstaltungen hat sich das Institut regional und überregional sowohl als Einrichtung insgesamt als auch mit Einzelprojekten präsentieren können, sei es bei Podiumsdiskussionen und Gesprächsveranstaltungen, bei Preisverleihungen oder Buchpräsentationen institutseigener Neuerscheinungen.

In den "Schriften zur sächsischen Geschichte und Volkskunde" konnten im Berichtsjahr drei Bände, in der Reihe der „Bausteine aus dem ISGV“ zwei Bände vorgelegt werden. In den Reihen „Quellen und Materialien zur sächsischen Geschichte und Volkskunde“ und "Spurensuche“ ist jeweils ein Band herausgekommen. Außerdem sind die beiden Zeitschriften des Instituts - das „Neue Archiv für sächsische Geschichte“ und die „Volkskunde in Sachsen“ - im gewohnten jährlichen Rhythmus erschienen. Neben den Redaktionsarbeiten an den gedruckten Veröffentlichungen hat auch die laufende Betreuung der Internet-Publikationen, nunmehr insgesamt neun laufende Vorhaben, im Berichtszeitraum wieder viel Arbeitskraft gebunden.

Die Erneuerung der EDV/IT-Infrastruktur des ISGV wurde im Berichtsjahr hardwie softwareseitig fortgesetzt. Die Erschließung der Bibliotheksbestände im OPAC wurde 2012 weiter optimiert. Die Angebote des Instituts werden im weltweiten Netz intensiv nachgefragt, was an 32.100 Besuchern mit 115.300 Seitenaufrufen auf der ISGV-Homepage im Berichtszeitraum ablesbar ist.

Im Personalbereich gab es bei den Haushaltsstellen durch Neuvergabe der Promotionsförderung eine Veränderung. Von den drittmittelfinanzierten Mitarbeitern sind die des „Sächsischen Klosterbuchs“ durch Auslaufen des Vorhabens ausgeschieden. Maike Günther, im ISGV mit der Planung des „Hauses der Archäologie und Geschichte Sachsens" in Chemnitz betraut, hat im Oktober 2012 eine Dauerstelle im Stadtgeschichtlichen Museum Leipzig angetreten. Petr Lozoviuk erhielt einen Ruf nach Pilzen und ist Ende Februar 2013 ausgeschieden. Die Geschäftsführung des Instituts lag im gesamten Berichtsjahr in den Händen des Leipziger Mitglieds des Direktoriums. 


\section{Forschungsprojekte 2012}

\section{Gemeinsame Projekte der Bereiche Geschichte und Volkskunde}

Schriften zur sächsischen Geschichte und Volkskunde; Quellen und Materialien zur sächsischen Geschichte und Volkskunde; Bausteine zur sächsischen Geschichte und Volkskunde; Spurensuche. Geschichte und Kultur Sachsens. Projektleiter: Direktorium/Bereichsleiter, Projektbearbeiter: Direktorium/Bereichsleiter/wissenschaftliche Mitarbeiter.

Beteiligung an dem internationalen Projekt „Entwicklung und Durchführung einer Konzeption für die Dauerausstellung zur Geschichte der Deutschen in den böhmischen Ländern“. Projektleiter am ISGV: Winfried Müller/Manfred Seifert, Projektverantwortlicher: Petr Lozoviuk.

Wandel ländlicher Freiraumstrukturen in Sachsen. Projektleiter: Catrin Schmidt/Erika Schmidt/Martina Schattkowsky/Manfred Seifert, Projektbearbeiter: Heiko Lieske/ Nadine Kulbe.

Sächsische Kulturlandschaften. Projektleiter: Enno Bünz/Winfried Müller/Martina Schattkowsky, Projektbearbeiter: Enno Bünz/Winfried Müller/Martina Schattkowsky.

\section{Projekte des Bereichs Geschichte}

Sächsische Biografie. Projektleiter: Martina Schattkowsky, Projektbearbeiter: Martina Schattkowsky/Frank Metasch/Lutz Vogel.

Fürstinnenkorrespondenzen der Reformationszeit, Teil 1: Die Briefe der Herzogin Elisabeth von Sachsen in der Zeit ihrer Rochlitzer Witwenschaft. Projektleiter: Martina Schattkowsky, Projektbearbeiter: Jens Klingner.

Codex diplomaticus Saxoniae. Das Urkundenbuch der Stadt Dresden. Projektleiter: Enno Bünz/Martina Schattkowsky, Projektbearbeiter: Ulrike Siewert.

Die Geschichte des Augustiner-Chorherrenstifts St. Afra in Meißen. Projektleiter: Enno Bünz, Projektbearbeiter: Dirk Martin Mütze.

Sächsisches Klosterbuch. Systematische Beschreibung der mittelalterlichen Klöster, Stifte und Komtureien im Gebiet des Freistaates Sachsen. Projektleiter: Enno Bünz, Projektbearbeiter: Dirk Martin Mütze/Sabine Zinsmeyer.

Ausstellungskonzeption für das „Haus der Archäologie und Geschichte Sachsens“. Projektleiter: ISGV (Direktorium, Bereichsleiter), Landesamt für Archäologie, Projektbearbeiter: Maike Günther, Projektmitarbeiter: Lutz Vogel.

Zwischen Migration und Assimilation. Adel im sächsisch-böhmischen Grenzraum (16./17. Jahrhundert). Projektleiter: Martina Schattkowsky, Projektbearbeiter: Martin Arnold. 
Für Gott und Vaterland - Patriotismus und Militärdienst in Sachsen 1806 bis 1866/67. Projektleiter: Winfried Müller, Projektbearbeiter: Torsten Schwenke.

Neues Archiv für sächsische Geschichte. Projektleiter: Karlheinz Blaschke/Enno Bünz/Winfried Müller/Martina Schattkowsky/Uwe Schirmer, Projektbearbeiter: Frank Metasch/Lutz Vogel.

\section{Projekte des Bereichs Volkskunde}

Lebensgeschichtliches Archiv für Sachsen. Projektleiter: Manfred Seifert, Projektbearbeiter: Sönke Friedreich.

Visuelle Quellen zur Volkskultur in Sachsen. Das Bildarchiv des ISGV. Projektleiter: Andreas Martin/Manfred Seifert, Projektbearbeiter: Andreas Martin.

Fremde - Heimat - Sachsen: Vertriebene als Neubauern. Staatliche Integrationsmaßnahmen und individuelle Adaptionsstrategien. Projektleiter: Manfred Seifert, Projektbearbeiter: Ira Spieker/Ursula Schlude/Sönke Friedreich.

An der Elbe. Das Leben mit dem Fluss. Projektleiter: Andreas Martin/Manfred Seifert, Projektbearbeiter: Andreas Martin.

Das Auge des Arbeiters. Untersuchungen zur proletarischen Amateurfotografie am Beispiel Sachsens. Projektleiter: Manfred Seifert, Projektbearbeiter: Wolfgang Hesse.

Lebensgeschichten aus der sächsisch-böhmischen Kontaktzone. Projektleiter: Petr Lozoviuk/Manfred Seifert, Projektbearbeiter: Petr Lozoviuk.

Das Eigene und das Fremde im Kontext des europäischen Modernisierungsprozesses im 19. Jahrhundert. Leipzig und Pressburg im Vergleich. Projektleiter: Manfred Seifert/Klaus Roth, Projektbearbeiter: Jan Schrastetter.

Informatisierung in der Landwirtschaft Sachsens. Projektleiter: Manfred Seifert, Projektbearbeiter: Birgit Huber.

Neue Sichtweisen. Zum Aufleben der Aussichtsturm-Begeisterung. Projektleiter: Andreas Martin/Manfred Seifert. Projektbearbeiter: Andreas Martin.

Künstlersteinzeichnungen für Haus und Schule: Die Produktion des Leipziger Wandschmuckverlags Merfeld \& Donner. Projektleiter: Winfried Müller, Projektbearbeiter: Winfried Müller/Silvio Dittrich.

Volkskunde in Sachsen. Projektleiter: ISGV, Projektbearbeiter: Manfred Seifert/Sönke Friedreich. 


\section{Mitwirkung an Ausstellungen und Ausstellungskonzeptionen}

Ausstellungskonzeption für das „Haus der Archäologie und Geschichte Sachsens“. Projektleiter: ISGV (Direktorium, Bereichsleiter), Landesamt für Archäologie, Projektbearbeiter: Maike Günther, Lutz Vogel.

Beteiligung an Entwicklung und Durchführung einer Konzeption für die Dauerausstellung zur Geschichte der Deutschen in den böhmischen Ländern. Projektleiter: Winfried Müller/Manfred Seifert, Projektbearbeiter: Petr Lozoviuk.

Zwischen Tradition und Modernität - Zeugnisse sächsischer Adelskultur (Arbeitstitel), Dauerausstellung, Schloss Nossen. Projektleiter: Martina Schattkowsky, Projektbearbeiter: Nicole Völtz/Martin Arnold.

Preußen und Sachsen. Szenen einer Nachbarschaft. Konzept/Organisation: Frank Göse (Universität Potsdam), Winfried Müller (ISGV), Anne-Katrin Ziesak (Haus der brandenburgisch-preußischen Geschichte, Potsdam).

Umsonst ist der Tod! Alltag und Frömmigkeit am Vorabend der Reformation. Konzept/Organisation: Hartmut Kühne (Berlin) in Zusammenarbeit mit den Kooperationspartnern; für das ISGV: Enno Bünz.

Beteiligung an der Sonderausstellung „Die königliche Jagdresidenz Hubertusburg und der Frieden von 1763“, Schloss Hubertusburg, 28. April bis 5. Oktober 2013. Konzeption/Organisation: Frank Metasch.

Wanderausstellung im Rahmen des Projekts „Fremde - Heimat - Sachsen: Vertriebene als Neubauern“. Konzeption/Organisation: Ira Spieker/Uta Bretschneider.

\section{Tagungen und Workshops 2012}

Adelslandschaft Mitteldeutschland. Die Rolle des landsässigen Adels in der mitteldeutschen Geschichte, Kloster Drübeck, 7. bis 9. März 2012. Konzeption/Organisation: Enno Bünz/Ulrike Höroldt (LHA Sachsen-Anhalt, Magdeburg)/Christoph Volkmar (LHA Sachsen-Anhalt, Wernigerode).

Visualisierte Minderheiten. Probleme und Möglichkeiten der musealen Präsentation von ethnischen bzw. nationalen Minderheiten in Zentraleuropa, Dresden, 30. bis 31. März 2012. Konzeption/Organisation: Petr Lozoviuk/Winfried Müller/Manfred Seifert.

Alltag und Frömmigkeit am Vorabend der Reformation in Mitteldeutschland, Leipzig, 19. bis 21. April 2012. Konzeption/Organisation: Enno Bünz/Hartmut Kühne (Berlin).

Die Elbe - Fluss ohne Grenzen, Dresden, 9. Juni 2012. Konzeption/Organisation: Andreas Martin.

Neue Forschungen zu sächsischen Klöstern. Ergebnisse und Perspektiven, Pirna, 26. bis 27. Oktober 2012. Konzeption/Organisation: Enno Bünz/Dirk Martin Mütze/ Sabine Zinsmeyer. 


\section{Publikationen 2012}

Neues Archiv für sächsische Geschichte, hrsg. von Karlheinz Blaschke/Enno Bünz/ Winfried Müller/Martina Schattkowsky/Uwe Schirmer, Redaktion: Frank Metasch (Schriftleitung)/Lutz Vogel (Rezensionen), Bd. 83 (2012), Neustadt an der Aisch: Verlag Ph. C. W. Schmidt.

Volkskunde in Sachsen, hrsg. vom Institut für Sächsische Geschichte und Volkskunde e. V., Schriftleitung: Manfred Seifert/Sönke Friedreich unter Mitarbeit von Wolfgang Hesse/Katrin Lehnert/Petr Lozoviuk/Andreas Martin/Ira Spieker, Bd. 24 (2012), Dresden: Thelem.

Schriften zur sächsischen Geschichte und Volkskunde, im Auftrag des Instituts für Sächsische Geschichte und Volkskunde e. V. hrsg. von Enno Bünz/Winfried Müller/ Martina Schattkowsky/Manfred Seifert, Leipzig: Leipziger Universitätsverlag:

Band 38 EnNo BüNZ (Hg.), 100 Jahre Landesgeschichte (1906-2006). Leipziger Leistungen, Verwicklungen und Wirkungen, 2012.

Band 39 Landschaft quer Denken. Theorien - Bilder - Formationen, hrsg. von Stefanie Krebs und Manfred Seifert unter Mitarbeit von Guido Fackler, Norbert Fischer, Andreas Martin und Miriam Volmert, 2012.

Band 41 Petr Lozoviuk, Grenzland als Lebenswelt. Grenzkonstruktionen, Grenzwahrnehmungen und Grenzdiskurse in sächsisch-tschechischer Perspektive, 2012.

Bausteine aus dem Institut für Sächsische Geschichte und Volkskunde. Kleine Schriften zur sächsischen Geschichte und Volkskunde, hrsg. von Enno Bünz/Winfried Müller/Martina Schattkowsky/Manfred Seifert, Dresden: Thelem:

Band 25 Petr Lozoviuk (Hg.), Ethnizität und Interethnik in der tschechischen Ethnologie, 2012.

Band 26 Petr Lozoviuk (Hg.), Visualisierte Minderheiten. Probleme und Möglichkeiten der musealen Präsentation von ethnischen bzw. nationalen Minderheiten, 2012.

Quellen und Materialien zur sächsischen Geschichte und Volkskunde, hrsg. von Enno Bünz/Winfried Müller/Martina Schattkowsky/Manfred Seifert, Leipzig: Leipziger Universitätsverlag:

Band 4 Katalog der Handschriften der Domstiftsbibliothek Bautzen, bearb. von Ulrike Spyra und Birgit Mitzscherlich unter Mitarbeit von Christoph Mackert und Agnes Scholla. Mit einer Einführung von Enno Bünz, 2012.

Spurensuche. Geschichte und Kultur Sachsens, im Auftrag des Instituts für Sächsische Geschichte und Volkskunde e. V. hrsg. von Enno Bünz/Winfried Müller/Martina Schattkowsky/Manfred Seifert, Dresden: Thelem:

Band 5 Andreas Martin/Anke Fröhlich, Die Flusslandschaft an den Mulden. Frühe Wahrnehmungen in bildender Kunst und Reiseliteratur, 2012. 


\section{Online-Publikationen (Weiterfübrung)}

Digitales Historisches Ortsverzeichnis von Sachsen. Wissenschaftliche Leitung: Ulrike Siewert, URL: http://hov.isgv.de/

Visuelle Quellen zur Volkskultur in Sachsen. Das Bildarchiv des ISGV, hrsg. vom Institut für Sächsische Geschichte und Volkskunde e. V. Wissenschaftliche Leitung: Andreas Martin, URL: http://bild.isgv.de/

Lebensgeschichtliches Archiv für Sachsen. Wissenschaftliche Leitung: Manfred Seifert, URL: http://ga.isgv.de/

Sächsische Biografie, hrsg. vom Institut für Sächsische Geschichte und Volkskunde e. V. Wissenschaftliche Leitung: Martina Schattkowsky, URL: http://www.isgv.de/ saebi/

Der Codex diplomaticus Saxoniae im Internet, hrsg. vom Institut für Sächsische Geschichte und Volkskunde e. V. Wissenschaftliche Leitung: Ulrike Siewert, URL: http://www.isgv.de/codex/

Repertorium Saxonicum, hrsg. vom Institut für Sächsische Geschichte und Volkskunde e. V. Wissenschaftliche Leitung: Ulrike Siewert, URL: http://www.isgv.de/ repsax/

Das Auge des Arbeiters. Untersuchungen zur proletarischen Amateurfotografie am Beispiel Sachsens. Wissenschaftliche Leitung: Wolfgang Hesse/Manfred Seifert, URL: http://www.arbeiterfotografie-sachsen.de/

Fremde - Heimat - Sachsen: Vertriebene als Neubauern. Staatliche Integrationsmaßnahmen und individuelle Adaptionsstrategien. Wissenschaftliche Leitung: Manfred Seifert/Ira Spieker/Sönke Friedreich, URL: http://www.neubauern-sachsen.de/

Sachsen.digital: Interdisziplinäre Wissensplattform zur Geschichte, Kultur und Landeskunde Sachsens. Gemeinsames Internetportal der Sächsischen Landesbibliothek Staats- und Universitätsbibliothek Dresden und des ISGV, URL: http://www.sachsendigital.de/ 